\title{
Comparative genomic analysis of toxin- negative strains of Clostridium difficile from humans and animals with symptoms of gastrointestinal disease
}

Piklu Roy Chowdhury ${ }^{1,2^{*}}$, Matthew DeMaere ${ }^{1}$, Toni Chapman ${ }^{2}$, Paul Worden ${ }^{1}$, lan G. Charles ${ }^{1,3}$, Aaron E. Darling ${ }^{1}$ and Steven P. Djordjevic ${ }^{1 *}$

\begin{abstract}
Background: Clostridium difficile infections (CDI) are a significant health problem to humans and food animals. Clostridial toxins ToxA and ToxB encoded by genes $t c d A$ and $t c d B$ are located on a pathogenicity locus known as the PaLoc and are the major virulence factors of $C$. difficile. While toxin-negative strains of $C$. difficile are often isolated from faeces of animals and patients suffering from CDI, they are not considered to play a role in disease. Toxin-negative strains of $C$. difficile have been used successfully to treat recurring CDI but their propensity to acquire the PaLoc via lateral gene transfer and express clinically relevant levels of toxins has reinforced the need to characterise them genetically. In addition, further studies that examine the pathogenic potential of toxin-negative strains of $C$. difficile and the frequency by which toxin-negative strains may acquire the PaLoc are needed.
\end{abstract}

Results: We undertook a comparative genomic analysis of five Australian toxin-negative isolates of $C$. difficile that lack $t c d A, t c d B$ and both binary toxin genes $c d t A$ and $c d t B$ that were recovered from humans and farm animals with symptoms of gastrointestinal disease. Our analyses show that the five $C$. difficile isolates cluster closely with virulent toxigenic strains of $C$. difficile belonging to the same sequence type (ST) and have virulence gene profiles akin to those in toxigenic strains. Furthermore, phage acquisition appears to have played a key role in the evolution of $C$. difficile.

Conclusions: Our results are consistent with the C. difficile global population structure comprising six clades each containing both toxin-positive and toxin-negative strains. Our data also suggests that toxin-negative strains of $C$. difficile encode a repertoire of putative virulence factors that are similar to those found in toxigenic strains of $C$. difficile, raising the possibility that acquisition of PaLoc by toxin-negative strains poses a threat to human health. Studies in appropriate animal models are needed to examine the pathogenic potential of toxin-negative strains of $\mathrm{C}$. difficile and to determine the frequency by which toxin-negative strains may acquire the PaLoc.

Keywords: Clostridium difficile, Toxin-negative isolates, Comparative genomics, CDI, Zoonosis

\footnotetext{
* Correspondence: Piklu.Bhattacharya@uts.edu.au;

Steven.Djordjevic@uts.edu.au

${ }^{1}$ The ithree institute, University of Technology Sydney, Sydney 2007, Australia

Full list of author information is available at the end of the article
} 


\section{Background}

Clostridium difficile is a Gram-positive pathogen that has emerged to become one of the leading causes of infectious diarrhoea in adult humans, securing its inclusion in the ESCAPE group of pathogens [1-4]. C. difficile infections range from being asymptomatic to causing mild or severe diarrhoea and occasionally lifethreatening conditions such as pseudomembranous colitis and toxic megacolon [1, 5]. However, communityacquired $C$. difficile infection is being reported with increasing frequency [6] and $C$. difficile is also emerging as a pathogen in animals particularly cattle, pigs and horses [5, 7-10]. Molecular epidemiological studies show that infections in humans and animals can share the same ribotype or multilocus sequence type (ST) [11] suggesting that pathogenic $C$. difficile may traffic between humans and animals, although further studies are needed to confirm these linkages.

C. difficile is a genetically diverse and globally dispersed species [11-16] having a clonal structure comprising six major clades (clades 1, 2, 3, 4, 5 and C-I). Clade $\mathrm{C}$-I is the most phylogenetically divergent clade and may represent of a new subspecies of $C$. difficile [17]. Clade C-I typically comprise toxin-negative strains of $C$. difficile [17] but toxigenic variants that reside in Clade C-I have recently been described [18]. Representatives from most clades have been associated with CDI in humans and comprise toxigenic strains with $\mathrm{A}^{+} / \mathrm{B}^{+}, \mathrm{A}^{-} / \mathrm{B}^{+}$ toxin types $[11,14,17,19-22]$. Non-toxigenic strains of $C$. difficile are represented in all six clades [11].

Toxin expression is considered mandatory for the development of $C$. difficile disease [23, 24]. Two large clostridial toxins known as toxins $\mathrm{A}(308 \mathrm{kDa})$ and $\mathrm{B}$ (260 kDa) encoded by $t c d A$ and $t c d B$ and the genes implicated in regulating their expression $(t c d C, t c d E$ and $t c d R$ ) reside on a $19.6-\mathrm{kb}$ pathogenicity locus known as the PaLoc $[25,26]$. The PaLoc is replaced by $115 / 75$ base pair non-coding region in toxin negative strains of $C$. difficile [27]. Approximately $20 \%$ of C. difficile strains express a third toxin, known as the binary toxin (CDT) [28]. Genes encoding binary toxin $(c d t A$ and $c d t B)$ and a regulator gene $(c d t R)$ are usually located on a locus (CdtLoc) that is physically separated from the PaLoc. A recent study described six toxin-negative (A-/B-) isolates of $C$. difficile that were positive for CDT from patient with symptoms of CDI [28].

Assays that detect toxin genes or the products of their expression dominate laboratory-based tests used to diagnose infections caused by $C$. difficile [29, 30]. Diagnostic tests that target $t c d$ genes underestimate the frequency of detection of toxin-negative strains (including those that express binary toxin) in $C$. difficile disease and as such, their role in disease is poorly understood. Phylogenetic studies show that toxin-negative strains of $C$. difficile cluster tightly with toxin-positive isolates belonging to the same ST [17] suggesting that presence and absence of the PaLoc may be one of the major defining features that differentiate toxin-negative strains from toxin producing strains of $C$. difficile. Notably, oral bacteriotherapy with toxin-negative strains or their spores has been used successfully to treat patients undergoing long-term antibiotic regimes and prevent colonisation by toxigenic strains of $C$. difficile [31-33]. The utility of this therapeutic strategy is supported by previous studies in hamsters which showed that exposure of the gastrointestinal tract to toxin-negative $C$. difficile strains prevented colonisation by toxin-positive strains [34, 35]. Interestingly, challenge studies in hamsters have shown that toxin-negative strains can effectively colonise the gut $[36,37]$ suggesting that toxin production may be of little consequence in determining the success of colonisation of the gastrointestinal tract. Notably, the toxin-negative strain CD1342 $\left(t c d A^{-}, t c d B^{-}\right.$, $c d t A^{-}$and $c d t B^{-}$) was reported to elicit an innate immune response in the caecum resulting in neutrophil infiltration, damage to epithelial mucosa and localised haemorrhagic congestion [36]. These findings suggest that virulence factors are carried by $C$. difficile in addition to the known toxins that can induce host pathology.

Studies of toxin-negative $C$. difficile strains have focused on the characterisation of functional binary toxins and their roles in pathogenesis [28, 33, 38]. The binary toxins $c d t A$ and $c d t B$ have adenosine diphosphate ribosyltransferase activity but their capacity to induce symptoms of $C$. difficile infection remains unclear [39-42]. Several adhesins, ECM-binding proteins, proteases, motility proteins, hydrolytic enzymes and other surface-associated proteins have been described in $C$. difficile and these factors are likely to contribute significantly to the establishment, progression and severity of $C$. difficile disease [11, 43]. Therefore, further studies are needed to examine the pathogenic potential of toxin-negative strains of $C$. difficile and to determine the frequency at which toxin-negative strains may acquire the PaLoc and express toxins.

Studies that seek to understand the evolutionary history of the PaLoc highlight the complex nature of the multiple clade-specific acquisitions that have occurred after clonal expansion of each clade in populations of $C$. difficile [17]. Those studies report homologous and sitespecific recombination events as having played an important role in the loss and gain of the PaLoc [17]. The PaLoc is proposed to be a mobile element that can transfer to toxin-negative strains rendering the recipient with the ability to produce clinically relevant concentrations of ToxA and ToxB [44]. Toxin-negative strains are 
purported to be ancestral to modern $C$. difficile but lateral genetic events complicate phylogenetic interpretation and alternate hypotheses have been proposed [17]. Genomic studies incorporating a greater diversity of toxin-negative strains of $C$. difficile are needed to shed light on their potential to cause disease.

\section{Methods}

\section{Isolation and culture of Clostridium difficile}

All C. difficile isolates analysed in this study (P29, 5.3, 19.3, 22.1, H3) were obtained from watery diarrhoea stool samples from their respective hosts (Additional file 1: Table S1). The porcine and equine $C$. difficile isolates analysed in this study were sourced in 2008 from different geographical locations in New South Wales, Australia. The porcine isolate P29 was isolated from a stool sample submitted by the veterinarian attending a piglet with severe but non-fatal diarrhoea. The equine isolate $\mathrm{H} 3$ was isolated from a live neonatal foal suffering from non-fatal watery diarrhoea. Stool samples were tested with PCR targeting major ETEC virulence genes [45] and common viruses known to cause diarrhoea in neonatal animals and were plated on blood agar plates to select for enteric pathogens. The stool specimens were initially tested for Escherichia coli, Clostridium perfringens and $C$. difficile using species-specific PCR primers [46]. Briefly, DNA was extracted from $500 \mu \mathrm{l}$ of stool sample using a FastDNA spin kit (QBiogene, California, USA) and used as a template for PCR using primers specific for $C$. difficile and $C$. perfringens $16 \mathrm{~S}$ rDNA [46], $t c d A$ and $t c d B$ genes (see below) and for $E$. coli [45]. To enrich for $C$. difficile $100 \mu \mathrm{l}$ of each faecal sample was added to $10 \mathrm{ml}$ cooked meat medium (TM0102 Oxoid Australia) and incubated anaerobically at $37{ }^{\circ} \mathrm{C}$ for $24 \mathrm{~h}$ using the anoxomat system (MART Microbiology B.B., The Netherlands).

Two hundred $\mu \mathrm{l}$ of culture samples that tested positive for $C$. difficile by PCR were transferred (from cooked meat media enrichment broth) into an Eppendorf tube and centrifuged $(10,000 \mathrm{rpm}, 5 \mathrm{~min})$. The pellet was resuspended in $1 \mathrm{ml}$ of absolute ethanol (room temperature, $2 \mathrm{~h}$ with periodic inversions), harvested by centrifugation (10,000 rpm, $5 \mathrm{~min})$, resuspended in brain heart infusion broth $(100 \mu \mathrm{l})$ and plated onto $C$. difficile selective agar (CC-BHIA + Taurocholate, PP2362 Oxoid Australia). Plates were incubated under anaerobic conditions at $37{ }^{\circ} \mathrm{C}$ for $24 \mathrm{~h}$. Colonies morphologically representing $C$. difficile from each plate were selected and sub-cultured onto CC-BHIA + Taurocholate until pure cultures were achieved.

\section{DNA extraction}

For routine PCR, template DNA was extracted with Chelex (BIO-RAD) from $2 \mathrm{ml}$ brain heart infusion broth cultures grown under anaerobic conditions at $37{ }^{\circ} \mathrm{C}$ for $48 \mathrm{~h}$. Briefly, cell pellets were obtained by centrifuging $(10,000 \mathrm{rpm}$ for $5 \mathrm{~min}) 200 \mu \mathrm{l}$ aliquots of liquid culture, washed $2 \times$ with $500 \mu \mathrm{l}$ of sterile water and resuspended in $200 \mu \mathrm{l}$ of $6 \%$ Chelex solution made in Tris-EDTA buffer (pH 7.5). The samples were incubated at $56^{\circ} \mathrm{C}$ for $20 \mathrm{~min}$, vortexed for $10 \mathrm{~s}$ and incubated at $100{ }^{\circ} \mathrm{C}$ for $8 \mathrm{~min}$. After incubation, the sample was immediately transferred to ice. One aliquot was stored at $4{ }^{\circ} \mathrm{C}$ for routine PCR tests while the other aliquots were archived at $-20^{\circ} \mathrm{C}$.

Sequencing-quality genomic DNA was prepared from $2 \mathrm{ml}$ brain heart infusion broth culture of isolates grown under anaerobic conditions at $37^{\circ} \mathrm{C}$ for $48 \mathrm{~h}$. The overnight culture was harvested by centrifugation (10,000 rpm for $10 \mathrm{~min})$, washed in sterile PBS and resuspended in $180 \mu \mathrm{l}$ of lysis buffer comprising $20 \mathrm{mM}$ Tris- $\mathrm{HCl}, \mathrm{pH}$ 8.0, 2 mM EDTA, $1.2 \%$ Triton $\mathrm{X}-100$ and lysozyme $\left(20 \mathrm{mg} \mathrm{ml}^{-1}\right)$ and incubated for $45 \mathrm{~min}$ at $37{ }^{\circ} \mathrm{C}$. DNA was isolated using a DNeasy ${ }^{\circ}$ Blood and Tissue Kit (Qiagen) by adhering to the manufacturer's instructions for the extraction of DNA from Gram-positive bacteria.

\section{PCR conditions}

C. difficile specific 16S rDNA primers, C.diff-F: 5'TTGAGCGATTTACTTCGGTAAAGA-3' and C.diff-R: 5'-CCATCCTGTACTGGCTCACCT-3' were used for identification and confirmation of $C$. difficile in enrichment as well as pure cultures. The presence of the tpi gene (encoding Triose Phosphate Isomerase), $t c d A$ gene (encoding Toxin $\mathrm{A}$ ) and $t c d B$ gene (encoding Toxin $\mathrm{B}$ ) were tested using previously published primer pairs. Conditions for PCR were as described previously [22] with minor modifications. Briefly, PCR was carried out in $25 \mu \mathrm{l}$ volumes containing $2 \mu \mathrm{l}$ of Chelex extracted DNA, $2.5 \mu \mathrm{l}$ of $10 \times$ PCR buffer, $1.5 \mathrm{mM}$ of $\mathrm{MgCl}_{2}, 1 \mathrm{mM}$ of each dATP, dGTP, dCTP and dDTP (Bioline, Australia), $0.5 \mu \mathrm{M}$ of each primer and $1 \mathrm{U}$ of BioRad Taq polymerase (Bioline, Australia). PCR cycling conditions consisted of an initial denaturation cycle $\left(2 \mathrm{~min}, 95^{\circ} \mathrm{C}\right)$ followed by $30 \mathrm{cy}-$ cles of denaturation $\left(94{ }^{\circ} \mathrm{C}, 1 \mathrm{~min}\right)$, annealing $\left(55^{\circ} \mathrm{C}\right.$, $1 \mathrm{~min}$ ) and extension $\left(72{ }^{\circ} \mathrm{C}, 2 \mathrm{~min}\right)$. The cycling process was completed with a final extension of $72{ }^{\circ} \mathrm{C}$ for $5 \mathrm{~min}$.

\section{Whole genome sequencing, data assembly and phylogenetic analysis}

Sequencing was performed at the Next Generation Sequencing facility within the ithree institute at the University of Technology Sydney using a bench top Illumina MiSeq ${ }^{\circ}$ sequencer and MiSeq V3 chemistry. Genomic DNA stocks shipped to the sequencing facility at concentrations between 1.8 and of $3.7 \mathrm{ng}^{-1} \mathrm{l}^{-1}$ were used as template for the preparation of sequencing libraries. The genomes were sequenced and assembled de novo using published protocols [47]. Raw 
data and assembled genome sequences were submitted in GenBank under the following Bio-project numbers, 5.3: PRJNA232267, 19.3: PRJNA239262, 22.1: PRJNA239264, P29: PRJNA239265 and H3: PRJNA238844.

PhyloSift was used to conduct a phylogenetic analysis of the five $C$. difficile genomes (P29, 5.3, 19.3, 22.1, H3) with nine closed $C$. difficile genomes including strains M120, CF5, M68, 2007855, BI1, CD196, R20291, ATCC43255 and CD630 available in the NCBI genome database on the 18th of December 2014 [48]. FigTree version 1.4.0 (http://tree.bio.ed.ac.uk/software/figtree/) was used to draw phylogenetic trees. Genome sequences of C. perfringens (ATCC13124), Clostridium botulinum (ATCC19397) and Clostridium tetani (E88) were included as outgroups in the analysis. To improve visual resolution of the evolutionary distances between test and reference strains of $C$. difficile the final figure was generated without the out-groups.

For reference-genome based phylogenetic inference, raw Illumina reads from all taxa were mapped to a single reference (strain CD630) using BWA-MEM (ver0.7.9a) (Li unpublished, github commit: 3efc33160c) and consensus sequences generated using the samtools/bcftools (ver0.1.19-96b5f2294a) tool-chain [49]. The complete set of consensus sequences were combined into a multiple sequence alignment. 1,216,986 alignment columns containing unresolved nucleotides $(\mathrm{N})$ were removed using Mothur (ver1.33.3) [50]. A total of 3,073,266 (72 \%) polymorphic and non-polymorphic sites were retained for further analysis. The inclusion of invariant sites has been demonstrated to improve accuracy of whole genome phylogeny [51]. Maximum likelihood phylogenetic inference was employed using RAxML (ver8.0.20) [52] with the following options: raxmlHPC-PTHREADSSSE3 - T 40 -f a -x 2136841 - 1486312 -N autoMRE -m GTRCAT. Inference was carried out under a general time reversible (GTR) substitution model with an infinite mixture model for substitutional heterogeneity (CAT), following the suggestion of the RAxML user guide for datasets of this size. The CAT approximation has been previously demonstrated to be an accurate and highly efficient alternative to Gamma-distributed rate heterogeneity on data sets with many taxa $(73-1663)$ $[53,54]$. Confidence in each clade of the Maximum Likelihood tree was estimated using the rapid bootstrap procedure [55] with automatic extended majority-rule criterion (100 bootstraps) and the resulting tree and bootstrap confidence estimates were visualized with FigTree version 1.4.0 (http://tree.bio.ed.ac.uk/software/figtree/).

\section{Multi locus sequence typing and comparative genomic analysis}

The online version of $C$. difficile PubMLST database (http://pubmlst.org/cdifficile/) was used to sequence type the isolates from the assembled genome sequences. The database was also exploited to locate certain genes of interest.

The online version of the RAST annotation server (http://rast.nmpdr.org/) [56] was used to annotate the genomes. The Classic RAST annotation scheme and FigFAM release 70 were used to predict genes $(5.3=$ RASTID 6666666.71923, 19.3 = RAST-ID 6666666.71924, H3 $=$ RAST-ID 6666666.72094, P29 = RAST-ID 1440056.4 and $22.1=$ RAST-ID 6666666.72093). Amino acid sequences corresponding to translated peptide products of all open reading frames predicted by RAST [57] from each of the five genomes were used in the 'all Vs all' homology search protocol deposited in the github repository as cRBLH (https://github.com/cerebis/crblh/ tree/v0.1). The protocol included clustering of predicted peptide sequences using a modified reciprocal best hit method, where simplicity was favoured for the apparent advantage in identifying orthogroups [58]. The all vs. all homology search was carried out with LAST [59-61] using runtime parameters (-T 1 -f 0 -e 100). The best hits were used to generate a directed graph with genes as vertices and best hits as edges. Unidirectional links between any two nodes were then pruned. Sets of disconnected subgraphs were then analysed for weak intra-cluster linkages, which likely represented overlap between partially homologous protein clusters. Each subgraph was subjected to modularity optimisation [62] and further decomposed until modularity scores of constituent elements fell below a given threshold (0.2). The nodes of the resulting subgraphs were then written out as protein clusters. Singletons defined as nodes without a single edge to any other were deemed unique/isolated genes.

Whole genome comparisons were performed using Mauve version 2.3.1 $[63,64]$ and iterative BLASTn analysis (http://blast.ncbi.nlm.nih.gov/Blast.cgi). Inter-isolate regions of interest identified from genome-wide comparisons using Mauve, BLASTp and protein clustering analyses were analysed further using iterative BLASTn and BLASTp searches. Figures of comparative genomic analysis, including comparisons of the PaLoc, were compiled using locally downloaded version of EasyFig version 2.1 [65].

The genome of the epidemic C. difficile CD630 strain was used in whole genome BLASTp analysis (in RAST) with our test $C$. difficile genomes to identify genes that have been correlated with pathogenicity. All genes deemed as candidate alternative virulence genes or genes for which the products could potentially confer pathogenic traits were individually interrogated using BLASTp and setting amino acid alignment cut off set to $100 \%$ of input query sequence to avoid any data extrapolation. 


\section{Results}

Toxin-negative $C$. difficile from animals and humans with clinical disease

The original stool samples and primary enrichment cultures of the stool samples tested negative for $C$. difficile toxins A and B. PCR assays using DNA from enrichment broths tested negative for enteric (other than $C$. difficile) and viral pathogens associated with neonatal diarrhoea. The porcine faecal sample was negative for the enterotoxigenic E. coli genes STa, STb and LT and C. perfringens and the disease symptomology did not correlate with viral disease as diagnosed by the attending veterinarian. Similarly, the foal sample was tested for E. coli, Salmonella enterica and rotavirus and none were detected. While gastrointestinal disease was most likely associated with the presence of toxin-negative $C$. difficile we cannot rule out the possibility that disease was caused by unculturable/unknown pathogens present in the gastrointestinal tract of these animals. Toxinnegative human $C$. difficile isolates 5.3, 19.3 and 22.1 were collected in the course of routine diagnostic tests for $C$. difficile-associated diarrhoea in patients presenting typical symptoms of the disease at a gastrointestinal clinic in Sydney, Australia in 2008.

Interrogation of the $C$. difficile PubMLST database confirmed that none of the toxin-negative isolates in our cohort (P29, H3, 5.3, 19.3, 22.1) had homologs of the known $C$. difficile PubMLST toxin genes $(t c d A, t c d B$, $c d t A$ and $c d t B$ ) confirming our initial diagnostic PCR data for toxin A and B genes (Additional file 1: Table S1). Isolates 5.3 (ST15), P29 (ST109) and H3 (ST29) were distinct from each other and from ST types of Australian isolates included in a recent phylogenetic study of C. difficile (Additional file 1: Table S1) [17].

\section{Phylogenetic analysis of toxin-negative isolates of $C$. difficile}

A study of the evolution of the $C$ difficile pathogenicity locus (PaLoc) identified an extremely divergent clade $\mathrm{C}$-I that exclusively comprised toxin-negative isolates predominantly of Australian origin [17]. A maximumlikelihood phylogenetic tree using a reference-based, whole genome alignment protocol (see methods section for protocol details) that incorporates both variant and invariant sites of the $C$. difficile genome sequences was used to verify the ancestry of our toxin-negative isolates. Our approach uses approximately $72 \%$ of the C. difficile genome for the analysis, considerably more than what was used in the original study [17]. All 73 genomes and the reference genome CD630 used in the previous study [17] as well as additional closed genomes of C. difficile (strains 2007855, ATCC43255, BI1, CF5, M68, M120 and R20291 from the GenBank database) were used in our initial phylogenetic analysis. A preliminary phylogenetic tree (Additional file 2: Figure S12) revealed that our genome-based phylogeny was largely congruent with that described in an earlier study [11] and clearly indicated that none of the five genomes that were the subject of our study clustered within the divergent clade C-I.

Strains that resided within clade C-I showed greater than $5 \%$ sequence divergence from the reference genome at homologous sites. Clades that have diverged more than $5 \%$ from the reference genome can be poorly resolved in workflows based on Illumina read mapping to the reference [51]. Given this and the uncertain ancestry of the isolates included in clade C-I in the Dingle et al. [11] study, members of clade C-I were excluded from the subsequent analysis based on a cohort of 86 strains depicted in Fig. 1. The branching order of the five clades in our phylogenetic tree was congruent with that reported earlier [17] with identical clustering of strains in the different sub-clades within each of the five clades. The five toxin-negative isolates from our study clustered with strains in Clades 1 (C. difficile 5.3, and H3) and 4 (C. difficile 19.3, 22.1 and P29) that are known to contain toxin-negative strains [17]. Our toxin-negative isolates (sourced both from animal and human sources) also clustered with strains of the same sequence type that included both toxin-positive and toxin-negative strains isolated from human clinical specimens in an earlier study [17].

Our genome sequences were assembled with a de novo assembler using A5 [48]. Prior to conducting a detailed analysis of the toxin-negative $C$. difficile isolates we identified the closest reference genome for tiling genomic scaffolds. A preliminary phylogeny generated using PhyloSift and FastTree (Additional file 2: Figure S2) indicated that $C$. difficile strain CF5 (toxin-positive ST86) was the most appropriate reference to order genomic scaffolds of isolates 19.3 (ST39), 22.1 (ST39) and P29 (ST109) while C. difficile strain 630 (toxin-positive, ST54 (PCR ribotype 012) was appropriate to order genomic scaffolds of isolates 5.3 (ST15) and H3 (ST29). Strain CF5 was isolated from a patient in Belgium in 1995 while CD630 is a highly virulent, multiple antibiotic resistant strain of $C$. difficile that caused pseudomembranous colitis in a human patient and later caused an epidemic of $C$. difficile infection in a Swiss hospital ward in 1982. All down-stream analyses of the genomes presented in this study were performed on genomic assemblies with scaffolds ordered to match the reference genomes.

\section{Homology based functional similarity in the toxin- negative isolates}

Initially a Progressive Mauve alignment performed (Additional file 2: Figure S3) on genome sequences of 


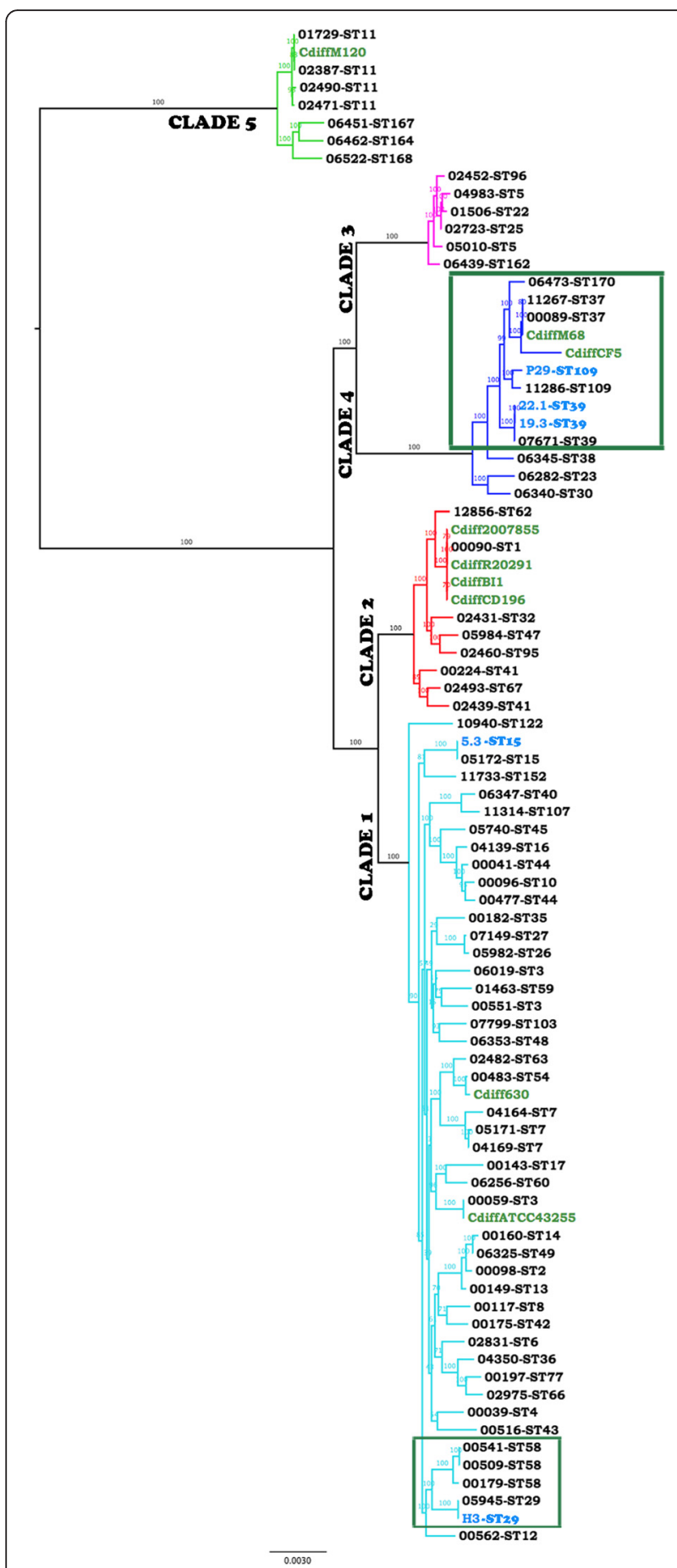

Fig. 1 A midpoint rooted phylogenetic tree depicting the five $C$. difficile clades. Australian toxin-negative strains are identified with blue strain names. Complete, closed genomes available in GenBank that were used as a reference are in green text. Genome names in black text are from a previous study [20]. The tree was constructed from reference-based whole genome alignments. Strains within boxed regions indicate genomes used in the ALL vs ALL BLASTp analysis. Clades highlighted in blue indicate clade 4 and in aqua is clade 1 human ST39 isolates 19.3 and 22.1 revealed a high level of nucleotide identity across the genomes with 324 SNP differences. Most of the SNPs were clustered into 7 groups (see Additional file 3: Table S4) suggesting that lateral gene transfer or homologous recombination-mediated genomic rearrangements may be responsible for the differences and were not considered further. Only 27 SNPs were identified that could generate changes in the amino acid sequence of the predicted proteins in the table Additional file 3: Table S4.

To identify genes affected by the SNP changes, a bidirectional BLASTp comparison of 3772 proteins comprising the predicted proteome of strain 19.3 was performed with 3764 predicted protein sequences from strain 22.1 in RAST. The analysis identified 3750 protein sequences that were identical in both the genomes. Nine protein sequences had greater than $99 \%$ sequence identity, six others had greater than $97 \%$ sequence identity and one ORF encoding a hypothetical protein showed 49 \% sequence identity (Additional file 3: Table S5). Proteins sharing 97 and $99 \%$ sequence identity predominantly encoded components of the bacterial cell surface including $\mathrm{N}$-acetylmuramoyl-L-alanine amidase, flagellar assembly protein $\mathrm{FliH}$, lipoprotein signal peptidase, putative $A B C$ transporters and permeases. Eight ORFs in isolate 19.3 predominantly encoding hypothetical proteins were missing in isolate 22.1. The genomes of isolates 19.3 and 22.1 comprise $4,181,809$ and 4,180,898 bp respectively.

Since isolates 22.1 and 19.3 had high levels of homology, predicted proteins only from isolate 19.3 were included in a pairwise bi-directional BLASTp analysis that seeks to identify conserved genes and major differences among toxin-negative isolates of Australian origin. Isolate P29 had the largest predicted proteome in our collection and was used as the reference. Comparisons of predicted proteomes of human isolates 5.3 \& 19.3 and equine isolate $\mathrm{H} 3$ with P29 identified major differences in regions harbouring prophage-associated proteins, hypothetical proteins (Additional file 3: Table S5) and putative transposases associated with mobile genetic elements.

\section{Chromosomal context of the PaLoc insertion site}

In toxin-negative strains, a 115 bp sequence replaces the PaLoc locus in phylogenetic clades 1 and 4 and the genetic context in which the $115 \mathrm{bp}$ sequence resides varies within the different clades that represent the $C$. difficile population structure [17]. An analysis of the genetic context of the $115 \mathrm{bp}$ sequence in our toxin-negative isolates compared to GenBank sequence entries representing chromosomal regions adjacent to the PaLoc insertion site in toxin-negative strains belonging to 
Clades 1 (GenBank Accession no HG002393) and 4 (GenBank Accession no HG002391) is depicted in Fig. 2. We identified several SNPs within the 115 bp-conserved fragment and an 80 nt long insertion in strains 5.3 and H3 downstream of the gene designated CD06642 in the reference genomes (Fig. 2). In addition, we identified a $68 \mathrm{nt}$ tandem repeat of the sequence adjacent to the 80 nt insertion site in strain H3 (see Additional file 2: Figure S7). Within members of clade 4, porcine isolate P29 had a deletion of the hypothetical gene seen in the reference region while human isolates 19.3 and 22.1 had a significant decrease in the nucleotide identity in hypothetical genes in the reference genome. Consistent with observations reported earlier, these differences indicate ongoing micro-evolutionary events within the locus that flanks the PaLoc insertion site [17].
Comparative BLASTp analyses of toxin-negative strains to identify functional similarities between groups of isolates An all versus all BLASTp based protein-clustering analysis was used to identify the putative core proteome of a subset of Clade 1 strains comprising five genomes and Clade 4 strains comprising 10 genomes (see boxed region in Fig. 1). To maintain uniformity in the input data used in the analysis, raw reads representing each of the genomes from an earlier study [17] were reassembled and annotated using the protocols that were used to assemble and annotate the five toxin-negative Australian isolates of $C$. difficile reported in this study, as described above. The assembly statistics and a comparison of the assembly outputs are presented in Additional file 3: Table S8. On an average, RAST predicted 3700 proteins per genome and these were included in the protein
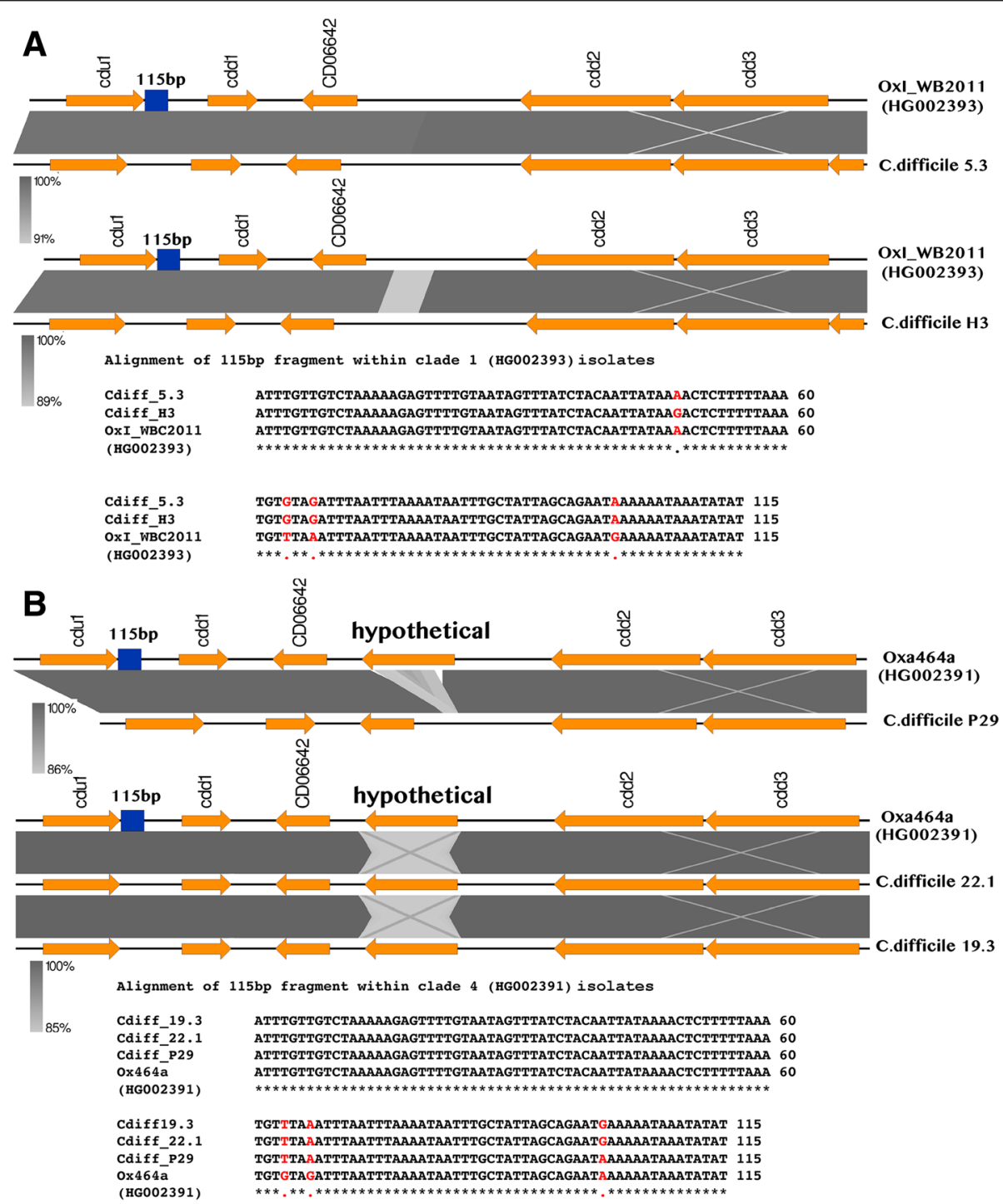

Fig. 2 Genetic context of the PaLoc in Australian toxin-negative strains of C. difficile. a Clade 1 prototype OxI_WB2011 (HG002393). b Clade 4 prototype Oxa464a (HG002391) 
clustering analysis. A bit score cut off of 50 was used to cluster homologous protein sequences. An overview of the data is presented in Table 1.

Ten clade 4 genomes boxed in Fig. 1 shared 3357 proteins (Table 1). Isolate P29 had the highest number (299) of unshared/unique proteins within the clade 4 cohort and most of the 299 proteins were phage-related (Additional file 3: Table S9). Some of the unique proteins clustered together in the same scaffold indicating lateral movement of phage-associated genomic DNA. C. difficile isolates 19.3 and 22.1 carried eight and seven unique proteins respectively. The handful of unshared proteins in 19.3 and 22.1 were attributed to mobile genetic elements or were designated to encode proteins of unknown function. The five $C$. difficile strains within clade 1 shared 3323 proteins. Equine isolate H3 had 111 unique proteins, most of which were phage related or hypothetical with some clustered in single scaffolds (Additional file 3: Table S9).

We queried the viral and prophage database in GenBank with the genome sequences of all five toxinnegative isolates using PHAST [66]. The database confirmed the presence of several regions contain phage

Table 1 Summary of protein clustering results within the different sub-clades containing the five toxin negative isolates included in this study. Summary of protein clusters within the different sub-clades of $C$. difficile

\begin{tabular}{ll}
\hline Clade 1 & No of predicted proteins \\
Genome names & 3323 \\
Core genome & \\
Total number of unique peptides in: & 45 \\
C. difficile C0000509 & 38 \\
C. difficile C0000541 & 176 \\
C. difficile C0000562 & 212 \\
C. difficile C00005945 & 111 \\
C. difficile H3 & \\
Clade 4: & 3357 \\
Core genome & \\
Total number of unique peptides in: & 8 \\
C. difficile 19.3 & 7 \\
C. difficile 22.1 & 33 \\
C. difficile C00000089 & 86 \\
C. difficile C000011286 & 84 \\
C. difficile C00006473 & 35 \\
C. difficile C00007671 & 60 \\
C. difficile C00011267 & 30 \\
C. difficile CF5 & 19 \\
C. difficile M68 & 299 \\
C. difficile P29 &
\end{tabular}

DNA in each of the five genomes in Clade 1 (see Additional file 2: Figure S10 and Additional file 4: Table S11). Table 2 lists a summary of the complete prophage sequences (PHAST scores $>110$ and over) identified in the five Australian toxin-negative isolates. Notably, isolates 19.3 and 22.1 returned identical phage profiles (Additional file 2: Figure S10). Both genomes carried an identical and complete $56.8 \mathrm{~kb}$ phage that is a close relative of phiC2, a $56.5 \mathrm{~kb}$ phage first identified in $C$. difficile strain $\mathrm{CD} 242$ [67]. phiC2 is one of the first completely sequenced temperate phages in $C$. difficile and regions of phiC2 are present in the majority of $C$. difficile genomes of clinical origin [68]. We also identified a phage in isolates P29 and H3 that carries sequence identity with phiC2. The prophage in P29 was larger $(97.4 \mathrm{~kb})$ than the original phiC2 sequence (Additional file 2: Figure S10). Isolate P29 also carries two other complete prophage sequences. One of these, prophage region 9 has significant sequence identity to the $C$. difficile temperate bacteriophage phiCD6356 that belongs to the Siphoviridae family [69]. The Siphoviridae family prophage identified in P29 is $52.8 \mathrm{~kb}$ (Additional file 4: Table S11) and is considerably larger than the first report of this phage sequence at $37.6 \mathrm{~kb}$ [69]. Isolate $\mathrm{H} 3$ also carries an identical variant of phage phiCD6356 (Additional file 2: Figure S12) comprising $41.1 \mathrm{~kb}$. Evidence of other complete prophage genomes is listed in Table 2 . Comparative BLASTp analysis of the four genomes (19.3, 5.3, H3 and P29) also aligned to the phage search protocol and confirmed the data generated by PHAST analyses, reinforcing the observation that the major differences in our Australian toxin-negative $C$. difficile isolates have a prophage origin.

Comparative BLASTp analysis of isolates 19.3, 5.3, H3 and P29 identified a $119.3 \mathrm{~kb}$ region on contig 11 in P29 (Additional file 5: Table S6). An all versus all protein clustering analysis also identified a subset of unique proteins on contig 11 of the P29 genome but not in the 10 human $C$. difficile genomes within clade 4 (Fig. 1). Equine isolate $\mathrm{H} 3$ was not included in this analysis as it was on a different clade. BLASTn analysis of the $119.3 \mathrm{~kb}$ region against the $C$. difficile genome database in GenBank identified similarity at the DNA level to parts of $C$. difficile strain CD630 indicating a phagemediated lateral movement of parts of the genome of CD630 into the genomes of isolates H3 and P29.

\section{Homology based functional prediction of Putative $C$. difficile virulence factors implicated in host colonization} A homology-based functional prediction analysis of proteins that have been experimentally verified, or predicted to play a role in the colonization of $C$. difficile strain CD630 with homologous proteins in the genomes of the toxin-negative isolates in our study is shown in Table 3 
Table 2 Summary of Phage related regions identified by PHAST in the 5 genomes. Phage sequences identified in this study

\begin{tabular}{|c|c|c|c|c|c|c|c|}
\hline $\begin{array}{l}\text { PHAST region } \\
\text { identifier }\end{array}$ & $\begin{array}{l}\text { Length of } \\
\text { prophage }\end{array}$ & $\begin{array}{l}\text { PHAST } \\
\text { score }\end{array}$ & $\begin{array}{l}\text { No of } \\
\text { predicted CDS }\end{array}$ & $\begin{array}{l}\text { Relative position } \\
\text { on genome }\end{array}$ & Predicted Phage & $\begin{array}{l}\mathrm{GC} \\
\text { content }\end{array}$ & $\begin{array}{l}\text { Location on Genomic } \\
\text { scaffolds }\end{array}$ \\
\hline \multicolumn{8}{|c|}{ C difficile P29 genome } \\
\hline Region_4 & $97.4 \mathrm{~Kb}$ & 150 & 126 & $1732045-1829500$ & PHAGE_Clostr_CDMH1_NC_024144 & 28.7 & $\begin{array}{l}27.1,30.1,40.1,34.1,36,1, \\
5.1,47.1 \text { and } 16.1\end{array}$ \\
\hline Region_8 & $113.6 \mathrm{~Kb}$ & 150 & 101 & $3773336-3886941$ & PHAGE_Geobac_virus_E2_NC_009552 & 40.8 & $5.1,19.1$ and 22.1 \\
\hline Region_9 & $52.8 \mathrm{~Kb}$ & 140 & 65 & $4233532-4286418$ & PHAGE_Clostr_phiCD6356_NC_015262 & 29.9 & $8.1,26.1,41.1$ and 42.1 \\
\hline Region_10 & $21.3 \mathrm{~Kb}$ & 100 & 22 & $4290994-4312349$ & PHAGE_Clostr_phiC2_NC_009231 & 36.4 & $\begin{array}{l}\text { In over } 20 \text { very small } \\
\text { scaffolds }\end{array}$ \\
\hline \multicolumn{8}{|c|}{ C. difficile $\mathrm{H} 3$ genome } \\
\hline Region_2 & $41.1 \mathrm{~Kb}$ & 150 & 63 & $925289-966449$ & PHAGE_Clostr_phiCD6356_NC_015262 & 28.5 & 22.1 and 31.1 \\
\hline Region_3 & $50.1 \mathrm{~Kb}$ & 110 & 66 & $1114600-1164714$ & PHAGE_Clostr_phiC2_NC_009231 & 28.1 & 18.1 and 5.1 \\
\hline Region_6 & $31.5 \mathrm{~Kb}$ & 110 & 47 & 4070500-4102080 & PHAGE_Clostr_phiC2_NC_009231 & 29.4 & $26.1,27.1,28.1,32.1,33.1$ \\
\hline \multicolumn{8}{|c|}{ C. difficile 5.3 genome } \\
\hline Region_3 & $57.9 \mathrm{~Kb}$ & 140 & 87 & $1555612-1613526$ & PHAGE_Clostr_phiC2_NC_009231 & 28.6 & 1.1 \\
\hline Region_4 & $45.1 \mathrm{~Kb}$ & 150 & 49 & $1741883-1787019$ & PHAGE_Clostr_phiSM101_NC_008265 & 27.2 & 1.1 \\
\hline \multicolumn{8}{|c|}{ C.difficle 19.3 genome } \\
\hline Region_3 & $56.8 \mathrm{~Kb}$ & 140 & 74 & 1700180-1757059 & PHAGE_Clostr_phiC2_NC_009231 & 28.8 & 1.1 \\
\hline \multicolumn{8}{|c|}{ C. difficile 22.1 genome: } \\
\hline Region_3 & $56.8 \mathrm{~Kb}$ & 140 & 74 & 1703858-1760737 & PHAGE_Clostr_phiC2_NC_009231 & 28.8 & 3.1 \\
\hline
\end{tabular}

$[70,71]$. Most of the proteins i had near perfect (98$100 \%)$ protein sequence identity including proteins encoded by $\operatorname{spoOA}$, which serves as a positive regulator for genes required in spore germination and groEL, a chaperone that also functions as an adhesin [72]. Several serine-proteases and other metalloproteases which may contribute to the disease development process [70] were also highly conserved. However, some membrane associated proteins that have been shown to play significant roles in the disease development process had lower alignment scores including SlpA, a surface layer protein that is proposed to facilitate host cell adherence [73] and FliC, an adhesin necessary for the colonization of gut epithelium [74].

\section{Discussion}

C. difficile colonisation in humans is age dependent. While asymptomatic carriage is common in infants less than three years of age it is rare in adults [75]. As such, infants can be a major reservoir of both pathogenic and toxin-negative strains in a community setting [75]. We isolated five toxin-negative isolates of $C$. difficile including three from humans $(22.1,19.3,5.3)$, one from a pig (P29) and one (H3) from a horse all showing symptoms of gastrointestinal disease. Despite efforts to identify $C$. difficile toxin genes or toxin gene products in the stool samples during the course of the isolation of these strains, none were detected. Phylogenetic studies showed that human ST39 isolates 19.3 and 22.1 and porcine
ST109 isolate P29 grouped with clinical human toxigenic strains of ST39 and ST109 respectively in Clade 4. Furthermore, human ST15 isolate 5.3 and equine ST29 isolate $\mathrm{H} 3$ grouped with human clinical toxigenic strains with ST15 and ST29 respectively in Clade 1. Comparative genome analyses showed that our toxin-negative isolates displayed virulence gene profiles akin to those identified in toxigenic strains. The animals from which samples were collected in this study exhibited gastrointestinal disease and we were unable to attribute these symptoms to the presence of toxin-positive strains of $C$. difficile. Given the mobility of the PaLoc [44] and evidence that the acquisition or loss of the PaLoc via recombination [17] has occurred multiple times during the evolution of the five major clades of $C$. difficile $[11,17]$, our data reinforces calls to include toxin-negative strains in genomic epidemiological studies of $C$. difficile $[17,36]$ and to better characterise asymptomatic carriage of closely related Clostridia in gut microbiome surveys such as the Human Microbiome Project and MetaHIT, both in humans and close animal contacts.

Our reference-based whole genome alignment and phylogeny analyses support the global population structure of C. difficile as described by Dingle et al. in 2014 $[14,17,19]$. Each clade has been shown previously to have representatives of both toxin-positive and toxinnegative strains [11]. Our toxin-negative isolates (19.3, 22.1, 5.3, P29, H3) belonged to STs that are distinct from those reported in an earlier study [17]. The role of toxins 
Table 3 Proteins derived from C. difficile CD630 that are predicted to play a role in pathogenesis

\begin{tabular}{|c|c|c|c|c|c|c|}
\hline \multirow[t]{2}{*}{ Selected gene and product } & \multirow{2}{*}{$\begin{array}{l}\text { C. difficile } 630 \\
\text { locus tag }\end{array}$} & \multirow{2}{*}{$\begin{array}{l}\text { Experimental } \\
\text { Verification }\end{array}$} & \multicolumn{4}{|l|}{ RAST Annotation identifiers } \\
\hline & & & In C. difficile 5.3 (\% identity) & In C. difficile 19.3 (\% identity) & In C. difficile H3 (\% identity) & In Cc difficile P29 (\% identity) \\
\hline Flagellin C gene flic & CD630_02390 & yes, RNAseq & fig|6666666.71923.peg.3142 (86) & fig|6666666.71924.peg.3067 (71)* & fig|6666666.72094.peg.3176 (87) & fig|1440056.4.peg.3191 (97) \\
\hline Flagellin $\mathrm{D}$ gene fliD & CD630_02370 & no & fig|6666666.71923.peg.3140 (88) & fig|6666666.71924.peg.3065 (61) & fig|6666666.72094.peg.3174 (88) & fig|1440056.4.peg.3193 (98) \\
\hline Precursor S-layer protein gene s/pA & CD630_27930 & yes, proteome & fig|6666666.71923.peg.2081 (43)* & fig|6666666.71924.peg.3725 (59) * & fig|6666666.72094.peg.2466 (58)* & fig|1440056.4.peg.2665 (54)* \\
\hline Stage 0 Sporulation gene spoA & CD630_12140 & yes, proteome & fig|6666666.71923.peg.158 (100) & fig|6666666.71924.peg.1561 (99) & fig|6666666.72094.peg.2932 (99) & fig|1440056.4.peg.240 (99) \\
\hline $\begin{array}{l}\text { Fibrinectin binding proten encoding } \\
\text { fbpA gene }\end{array}$ & CD630_25920 & no & fig|6666666.71923.peg.2930 (99) & fig|6666666.71924.peg.2028 (98) & fig|6666666.72094.peg.3740 (99) & fig|1440056.4.peg.41 (98) \\
\hline GroEL encoding gene grol & CD630_01940 & yes, proteome & fig|6666666.71923.peg.3095 (100) & fig|6666666.71924.peg.2995 (99) & fig|6666666.72094.peg.3129 (100) & fig|1440056.4.peg.3232 (99) \\
\hline Cell surface protein cwp66 & CD630_27890 & no & fig|6666666.71923.peg.2085 (60) & fig|6666666.71924.peg.3721 (78) & fig|6666666.72094.peg.2462 (77) & fig|1440056.4.peg.2669 (79) \\
\hline Protease cwp84 & CD630_27870 & no & fig|6666666.71923.peg.2087 (98) & fig|6666666.71924.peg.3719 (99) & fig|6666666.72094.peg.2460 (99) & fig|1440056.4.peg.2671 (99) \\
\hline Adhesin (LPXTG) & CD630_28310 & no & fig|6666666.71923.peg.2041 (99) & fig|6666666.71924.peg.3767 (94) & fig|6666666.72094.peg.2504 (98) & fig|1440056.4.peg.2625 (94) \\
\hline Cell wall binding protein encoding cwp2 & CD630_27910 & yes, proteome & fig|6666666.71923.peg.2083 (98) & fig|6666666.71924.peg.3723 (99) & fig|6666666.72094.peg.2464 (99) & fig|1440056.4.peg.2667 (99) \\
\hline Cell wall binding protein encoding cwp 12 & CD630_27940 & no & fig|6666666.71923.peg.2080 (65)* & fig|6666666.71924.peg.3726 (98) & fig|6666666.72094.peg.2467 (95) & fig|1440056.4.peg.2664 (94) \\
\hline Cell wall binding protein encoding cwp 11 & CD630_27950 & yes, proteome & fig|6666666.71923.peg.2079 (98) & fig|6666666.71924.peg.3727 (99) & fig|6666666.72094.peg.2468 (99) & fig|1440056.4.peg.2663 (99) \\
\hline Cell wall binding protein encoding cwp 9 & CD630_27980 & no & fig|6666666.71923.peg.2076 (99) & fig|6666666.71924.peg.3730 (99) & fig|6666666.72094.peg.2471 (99) & fig|1440056.4.peg.2660 (99) \\
\hline Cell wall hydrolase (LPXTG) & CD630_01830 & no & fig|6666666.71923.peg.3084 (97)* & fig|6666666.71924.peg.2984 (99) & fig|6666666.72094.peg.3118 (100) & fig|1440056.4.peg.3243 (99) \\
\hline $\begin{array}{l}\text { Cell wall binding protein encoding cwp } 25 \\
\text { gene }\end{array}$ & CD630_08440 & no & fig|6666666.71923.peg.2189 (100) & fig|6666666.71924.peg.3292 (97) & fig|6666666.72094.peg.2128 (99) & fig|1440056.4.peg.522 (97) \\
\hline $\begin{array}{l}\text { N-acetylmuramoyl-L-analini amidase } \\
\text { encoding cwp16 }\end{array}$ & CD630_10350 & no & fig|6666666.71923.peg.1 (99) & fig|6666666.71924.peg.3716 (65)* & fig|6666666.72094.peg.1495 (99) & fig|1440056.4.peg.1845 (98) \\
\hline $\begin{array}{l}\text { Cell wall hydrolase encoding gene } \\
\text { (invasin) }\end{array}$ & CD630_27680 & no & fig|6666666.71923.peg.2107 (99) & fig|6666666.71924.peg.3700 (99) & fig|6666666.72094.peg.2441 (99) & fig|1440056.4.peg.2690 (99) \\
\hline Polysaccharide de-acetylase & CD630_15220 & $\begin{array}{l}\text { yes, RNAseq } \\
\text { and proteome }\end{array}$ & fig|6666666.71923.peg.489 (100) & fig|6666666.71924.peg.291 (99) & fig|6666666.72094.peg.3592 (100) & fig|1440056.4.peg.1197 (99) \\
\hline LmbE-like deacetylase encoding gene & CD630_27900 & no & fig|6666666.71923.peg.2084 (93) & fig|6666666.71924.peg.3722 (100) & fig|6666666.72094.peg.2463 (100) & fig|1440056.4.peg.2668 (97) \\
\hline $\begin{array}{l}\text { Invasin/Sh3 domain containing surface } \\
\text { protein }\end{array}$ & CD630_11350 & no & fig|6666666.71923.peg.77 (100) & fig|6666666.71924.peg.1641 (98) & fig|6666666.72094.peg.2849 (99) & fig|1440056.4.peg.320 (98) \\
\hline $\begin{array}{l}\text { Cell wall hydrolase/Invasin associated } \\
\text { protein }\end{array}$ & CD630_24020 & & fig|6666666.71923.peg.2730 (100) & fig|6666666.71924.peg.2779 (98) & fig|6666666.72094.peg.2094 (99) & fig|1440056.4.peg.3691 (99) \\
\hline $\begin{array}{l}\text { Autolysin acd gene homolog/mannosyl- } \\
\text { glycoprotein endo neta } \mathrm{N} \text { acetylglucosamine }\end{array}$ & CD630_13040 & no & fig|6666666.71923.peg.256 (100) & fig|6666666.71924.peg.1460 (98) & fig|6666666.72094.peg.3031 (99) & fig|1440056.4.peg.158 (98) \\
\hline Protease/Serine protease, HrtA family & CD630_32840 & no & fig|6666666.71923.peg.1608 (100) & fig|6666666.71924.peg.3459 (99) & fig|6666666.72094.peg.64 (100) & fig|1440056.4.peg.2801 (99) \\
\hline Intracellular serine protease & CD630_32540 & no & fig|6666666.71923.peg.1638 (100) & fig|6666666.71924.peg.3426 (99) & fig|6666666.72094.peg.94 (99) & fig|1440056.4.peg.2834 (99) \\
\hline Protease/Subtilase family & CD630_07030 & no & fig|6666666.71923.peg.2324 (100) & fig|6666666.71924.peg.3155 (97) & fig|6666666.72094.peg.2691 (100) & fig|1440056.4.peg.1169 (97) \\
\hline $\begin{array}{l}\text { Ser-type protease/subtilisin-like serine } \\
\text { germination related protease }\end{array}$ & CD630_22470 & $\begin{array}{l}\text { yes, Mass } \\
\text { spectrometry }\end{array}$ & fig|6666666.71923.peg.1327 (99) & fig|6666666.71924.peg.2513 (99) & fig|6666666.72094.peg.1937 (99) & fig|1440056.4.peg.1500 (98) \\
\hline $\begin{array}{l}\text { Serine protease precursor/Subtilinase } \\
\text { subfamily }\end{array}$ & CD630_20000 & no & fig|6666666.71923.peg.1085 (100) & fig|6666666.71924.peg.2267 & fig|6666666.72094.peg.1687 (99) & fig|1440056.4.peg.2329 (99) \\
\hline $\begin{array}{l}\text { Membrane-associated zinc metalloprotease/ } \\
\text { M50 family peptidase }\end{array}$ & CD630_21290 & no & fig|6666666.71923.peg.1209 (100) & fig|6666666.71924.peg.2404 (99) & fig|6666666.72094.peg.1813 (100) & fig|1440056.4.peg.1610 (100) \\
\hline Zinc Protease/M16 family peptidase & CD630_26610 & yes, proteome & fig|6666666.71923.peg.2996 (100) & fig|6666666.71924.peg.626 (99) & fig|6666666.72094.peg.3677 (100) & fig|1440056.4.peg.438 (99) \\
\hline
\end{tabular}

* indicates gaps in alignment of amino acid sequences with reference, likely suggesting presence of inactive proteins or variants in the test genomes 
in $C$. difficile infection has been extensively studied but factors that enable $C$. difficile to efficiently colonise the human gastrointestinal tract are relatively poorly understood and are not associated with genes encoded on the PaLoc. It is not known why some toxigenic strains evolve into dominant hypervirulent clones. Thus, considering the genetic diversity inherent within the phylogenetic structure of $C$. difficile [14] a sub-population of toxin-negative strains of $C$. difficile that are efficient colonisers of the host gastrointestinal tract may readily acquire the PaLoc and evolve to become future hypervirulent strains. Several proteins have been suggested to play crucial roles in the colonization of gastrointestinal epithelium and disease progression [43, 71, 73, 74, 76-78]. A recent global proteome study of C. difficile strains CD630 and R20291 has identified numerous extracellular proteins from culture supernatants that may contribute to the virulence attributes of these strains [70].

Our study reinforced the important role played by phage in the evolution of $C$. difficile. While PHAST analysis was useful for identifying phage sequences, the analysis may not have identified the full extent of lysogenic phage because our draft genomes remain in multiple scaffolds. Although the complete sequence of phage phiC2 was identified in isolates 19.3 and 22.1 the regions that had significant homology with phiC2 in isolates P29 and $\mathrm{H} 3$ were located on different scaffolds. We used a scaffold tiling approach against the closed genome of a reference strain to create the input file for PHAST analysis (PHAST converts the scaffolded genomes into a concatenated artificial chromosome prior to predicting the phage content) and as such it remains a possibility that the partial matches are a consequence of the data handling process. Phage phiC2 is present in the majority of human isolates of $C$. difficile [68]. However, we detected regions of phiC2 in strains $\mathrm{P} 29$ and $\mathrm{H} 3$ suggesting that further studies are needed to address issues surrounding the association of phiC2 in C. difficile of animal origin. We also identified the $C$. difficile temperate bacteriophage phiCD6356 from the Siphoviridae family in isolates P29 and H3 but not in our human isolates of $C$. difficile. Genomes of bacteriophages belonging to the Siphoviridae family range in size from 14 to $50 \mathrm{~kb}[79,80]$ and this broad range may be a reflection of the stringency governing the amount of DNA that can be packaged by phiCD6356. In addition to the acquisition of phage-associated genes, a 119.3-kb region on contig 11 in isolate P29 was also identified in the course of this analysis. This region is unique to the P29 genome and displayed significant DNA sequence identity to portions of the CD630 genome. It remains unknown if the 119.3-kb region exists in C. difficile strains of porcine origin. Further analyses with greater numbers of genomes from both human and animal sources are required to conclusively address these questions.

\section{Conclusions}

Our studies reinforce calls to improve our understanding of the physiological conditions that promote lateral transfer of the PaLoc in the gastrointestinal tract [44]. This is important because the conditions that facilitate movement of fragments of DNA carrying the PaLoc and their recombination into the chromosome are also conducive to the movement of conjugative transposons that carry antibiotic resistance genes and putative virulence factors as independent genetic events [44].

\section{Data accessibility}

Genome sequences reported in this analysis were submitted to GenBank and are available via the accession numbers provided. The bioinformatics softwares are made available through the GitHub repository links.

\section{Additional files}

Additional file 1: Table S1. Isolation history, genomic assembly and initial PCR screening results of the five isolates analysed in this manuscript. (DOCX $36 \mathrm{~kb}$ )

Additional file 2: Figure S2. Preliminary phylogeny with reference genomes to identify the most closely related reference genome for tiling of genomic scaffolds. Figure S3. Mauve alignments of genomes 19.3 and 22.1. Figure S7. DNA sequence alignment of repetitive regions in PaLoc of isolates in clade 1. Figure S10. Phage profiles in the genomes of the five Australian toxin-negative $C$. difficile isolates included in this study. Figure S12. Preliminary Phylogenetic tree with all isolates included in Dingle et al's publication in 2011 [11]. Isolates that form clade C-I is highlighted in blue. (DOCX 2963 kb)

Additional file 3: Table S4. SNPs identified by Mauve alignments of genomes of C. difficile strains 19.3 and 22.1. Table S5. Bidirectional BLASTp analysis of peptide sequences predicted from genomes of C. difficile strains 19.3 and 22.1 to identify amino acid changes caused by SNPs in the genomes. Table S8. Assembly Statistics downloaded from short read archives. Table S9. Unique proteins identified from the All Vs All BLASTp analysis of C. difficile genomes. (XLSX $632 \mathrm{~kb}$ )

Additional file 4: Table S11. Regions identified in each C. difficile genome by PHAST. Sheet 1. C. difficile strain P29 genome, Sheet 2. C. difficile strain H3 genome, Sheet 3. C. difficile strain 5.3 genome, Sheet 4. C. difficile strain 19.3 genome. Sheet 5. C. difficile strain 22.1 genome. (XLSX $134 \mathrm{~kb}$ )

Additional file 5: Table S6. Sheet $1=$ Comparative BLASTp analysis of 4 genomes, Sheet 2 = Alignment of scaffold 11.1 of genome P29 against $C$. difficile CD630 genome. (XLSX 682 kb)

\section{Competing interests}

The authors declare that they have no competing interests, including financial competing interests.

\section{Authors' contribution}

PRC assembled the genome sequences, conceived, analysed and designed the presentation of data in the manuscript, performed all comparative genomic analysis and benchwork to confirm features identified from the comparative genomic analysis and drafted the manuscript. AED and MD wrote data analysis software packages and scripts and assisted with data interpretation. TC cultured the isolates, performed PCR analyses for toxin genes strains, ribotyping and fingerprinting assays and designed aspects the 
project. PW constructed the libraries for genome sequencing. IGC assisted with experimental design, data analysis and writing the manuscript. SPD conceived and designed aspects of the project, managed the project and drafted the final manuscript. All authors read and approved the final manuscript.

\section{Acknowledgements}

This work is a product of the ausgem partnership. The authors wish to acknowledge Prof Thomas Borody for kindly providing the human isolate included in this analysis.

\section{Author details}

${ }^{1}$ The ithree institute, University of Technology Sydney, Sydney 2007, Australia. ${ }^{2}$ NSW Department of Primary Industries, Elizabeth Macarthur Agricultural Institute, PMB 8, Camden, NSW 2570, Australia. Institute of Food Research, Norwich Research Park, Colney, Norwich NR4 7UA, UK.

Received: 29 October 2015 Accepted: 2 March 2016 Published online: 12 March 2016

\section{References}

1. Karadsheh Z, Sule S. Fecal transplantation for the treatment of recurrent clostridium difficile infection. N Am J Med Sci. 2013:5(6):339-43. Pubmed Central PMCID: 3731863

2. Lessa FC, Mu Y, Bamberg WM, Beldavs ZG, Dumyati GK, Dunn JR, et al. Burden of Clostridium difficile infection in the United States. N Engl J Med. 2015:372(9):825-34

3. Peterson LR. Bad bugs, no drugs: no ESCAPE revisited. Clin Infect Dis. 2009; 49(6):992-3.

4. Redelings MD, Sorvillo F, Mascola L. Increase in Clostridium difficile-related mortality rates, United States, 1999-2004. Emerg Infect Dis. 2007;13(9): 1417-9. Pubmed Central PMCID: 2857309.

5. Rupnik M, Wilcox MH, Gerding DN. Clostridium difficile infection: new developments in epidemiology and pathogenesis. Nat Rev Microbiol. 2009; 7(7):526-36.

6. Khanna S, Pardi DS, Aronson SL, Kammer PP, Baddour LM. Outcomes in community-acquired Clostridium difficile infection. Aliment Pharmacol Ther. 2012:35(5):613-8. Pubmed Central PMCID: 3293482

7. Bauer MP, Kuijper EJ. Potential sources of Clostridium difficile in human infection. Infect Dis Clin North Am. 2015;29(1):29-35.

8. Songer JG, Anderson MA. Clostridium difficile: an important pathogen of food animals. Anaerobe. 2006:12(1):1-4.

9. Hensgens MP, Keessen EC, Squire MM, Riley TV, Koene MG, de Boer E, et al. Clostridium difficile infection in the community: a zoonotic disease? Clin Microbiol Infect. 2012;18(7):635-45.

10. Goorhuis A, Debast SB, van Leengoed LA, Harmanus C, Notermans DW, Bergwerff AA, et al. Clostridium difficile PCR ribotype 078: an emerging strain in humans and in pigs? J Clin Microbiol. 2008;46(3):1157. Pubmed Central PMCID: 2268365, author reply 8.

11. Dingle KE, Griffiths D, Didelot X, Evans J, Vaughan A, Kachrimanidou M, et al. Clinical Clostridium difficile: clonality and pathogenicity locus diversity. PLoS One. 2011;6(5):e19993. Pubmed Central PMCID: 3098275.

12. Walk ST, Micic D, Jain R, Lo ES, Trivedi I, Liu EW, et al. Clostridium difficile ribotype does not predict severe infection. Clin Infect Dis. 2012;55(12): 1661-8. Pubmed Central PMCID: 3501335

13. Cairns MD, Stabler RA, Shetty N, Wren BW. The continually evolving Clostridium difficile species. Future Microbiol. 2012;7(8):945-57.

14. Stabler RA, Dawson LF, Valiente E, Cairns MD, Martin MJ, Donahue EH, et al. Macro and micro diversity of Clostridium difficile isolates from diverse sources and geographical locations. PLoS One. 2012;7(3):e31559. Pubmed Central PMCID: 3292544

15. Behroozian AA, Chludzinski JP, Lo ES, Ewing SA, Waslawski S, Newton DW et al. Detection of mixed populations of Clostridium difficile from symptomatic patients using capillary-based polymerase chain reaction ribotyping. Infect Control Hosp Epidemiol. 2013;34(9):961-6. Pubmed Central PMCID: 4016961

16. Waslawski S, Lo ES, Ewing SA, Young VB, Aronoff DM, Sharp SE, et al. Clostridium difficile ribotype diversity at six health care institutions in the United States. J Clin Microbiol. 2013;51(6):1938-41. Pubmed Central PMCID: 3716112.
17. Dingle KE, Elliott B, Robinson E, Griffiths D, Eyre DW, Stoesser N, et al. Evolutionary history of the Clostridium difficile pathogenicity locus. Genome Biol Evol. 2014;6(1):36-52. Pubmed Central PMCID: 3914685

18. Monot M, Eckert C, Lemire A, Hamiot A, Dubois T, Tessier C, et al. Clostridium difficile: New insights into the evolution of the pathogenicity locus. Sci Rep. 2015:5:15023. Pubmed Central PMCID: 4597214.

19. Griffiths D, Fawley W, Kachrimanidou M, Bowden R, Crook DW, Fung R, et al. Multilocus sequence typing of Clostridium difficile. J Clin Microbiol. 2010; 48(3):770-8. Pubmed Central PMCID: 2832416.

20. Lemee L, Bourgeois I, Ruffin E, Collignon A, Lemeland JF, Pons JL. Multilocus sequence analysis and comparative evolution of virulence-associated genes and housekeeping genes of Clostridium difficile. Microbiology. 2005:151(Pt 10):3171-80.

21. Lemee L, Dhalluin A, Pestel-Caron M, Lemeland JF, Pons JL. Multilocus sequence typing analysis of human and animal Clostridium difficile isolates of various toxigenic types. J Clin Microbiol. 2004;42(6):2609-17. Pubmed Central PMCID: 427854

22. Lemee L, Dhalluin A, Testelin S, Mattrat MA, Maillard K, Lemeland JF, et al. Multiplex PCR targeting tpi (triose phosphate isomerase), tcdA (Toxin A), and tcdB (Toxin B) genes for toxigenic culture of Clostridium difficile. J Clin Microbiol. 2004:42(12):5710-4. Pubmed Central PMCID: 535266

23. Rupnik M. How to detect Clostridium difficile variant strains in a routine laboratory. Clin Microbiol Infect. 2001;7(8):417-20.

24. Voth DE, Ballard JD. Clostridium difficile toxins: mechanism of action and role in disease. Clin Microbiol Rev. 2005;18(2):247-63. Pubmed Central PMCID: 1082799.

25. Hundsberger $T$, Braun $V$, Weidmann $M$, Leukel $P$, Sauerborn $M$, von Eichel-Streiber C. Transcription analysis of the genes tcdA-E of the pathogenicity locus of Clostridium difficile. Eur J Biochem/FEBS. 1997; 244(3):735-42.

26. Matamouros S, England P, Dupuy B. Clostridium difficile toxin expression is inhibited by the novel regulator TcdC. Mol Microbiol. 2007;64(5):1274-88.

27. Braun V, Hundsberger T, Leukel P, Sauerborn M, von Eichel-Streiber C. Definition of the single integration site of the pathogenicity locus in Clostridium difficile. Gene. 1996;181(1-2):29-38.

28. Eckert C, Emirian A, Le Monnier A, Cathala L, De Montclos H, Goret J, et al. Prevalence and pathogenicity of binary toxin-positive Clostridium difficile strains that do not produce toxins A and B. New Microbes New Infect. 2015; 3:12-7. Pubmed Central PMCID: 4337936.

29. Collins DA, Elliott B, Riley TV. Molecular methods for detecting and typing of Clostridium difficile. Pathology. 2015;47(3):211-8.

30. Rupnik M, Brazier JS, Duerden BI, Grabnar M, Stubbs SL. Comparison of toxinotyping and PCR ribotyping of Clostridium difficile strains and description of novel toxinotypes. Microbiology. 2001;147(Pt 2):439-47.

31. Villano SA, Seiberling M, Tatarowicz W, Monnot-Chase E, Gerding DN. Evaluation of an oral suspension of VP20621, spores of nontoxigenic Clostridium difficile strain M3, in healthy subjects. Antimicrob Agents Chemother. 2012;56(10):5224-9. Pubmed Central PMCID: 3457387

32. Nagaro KJ, Phillips ST, Cheknis AK, Sambol SP, Zukowski WE, Johnson S, et al. Nontoxigenic Clostridium difficile protects hamsters against challenge with historic and epidemic strains of toxigenic BI/NAP1/027 C. difficile. Antimicrob Agents Chemother. 2013;57(11):5266-70. Pubmed Central PMCID: 3811292.

33. Natarajan M, Walk ST, Young VB, Aronoff DM. A clinical and epidemiological review of non-toxigenic Clostridium difficile. Anaerobe. 2013:22:1-5. Pubmed Central PMCID: 3729612.

34. Seal D, Borriello SP, Barclay F, Welch A, Piper M, Bonnycastle M. Treatment of relapsing Clostridium difficile diarrhoea by administration of a nontoxigenic strain. Eur J Clin Microbiol. 1987;6(1):51-3.

35. Wilson KH, Sheagren JN. Antagonism of toxigenic Clostridium difficile by nontoxigenic C. difficile. J Infect Dis. 1983;147(4):733-6.

36. Buckley AM, Spencer J, Maclellan LM, Candlish D, Irvine JJ, Douce GR. Susceptibility of hamsters to Clostridium difficile isolates of differing toxinotype. PLoS One. 2013;8(5):e64121. Pubmed Central PMCID: 3660315.

37. Sambol SP, Merrigan MM, Tang JK, Johnson S, Gerding DN. Colonization for the prevention of Clostridium difficile disease in hamsters. J Infect Dis. 2002; 186(12):1781-9.

38. Hung YP, Lin HJ, Wu TC, Liu HC, Lee JC, Lee Cl, et al. Risk factors of fecal toxigenic or non-toxigenic Clostridium difficile colonization: impact of Tolllike receptor polymorphisms and prior antibiotic exposure. PLoS One. 2013; 8(7):e69577. Pubmed Central PMCID: 3723847 
39. Gerding DN, Johnson S, Rupnik M, Aktories K. Clostridium difficile binary toxin CDT: mechanism, epidemiology, and potential clinical importance. Gut Microbes. 2014;5(1):15-27. Pubmed Central PMCID: 4049931.

40. Geric B, Carman RJ, Rupnik M, Genheimer CW, Sambol SP, Lyerly DM, et al. Binary toxin-producing, large clostridial toxin-negative Clostridium difficile strains are enterotoxic but do not cause disease in hamsters. J Infect Dis. 2006;193(8):1143-50.

41. Bacci S, Molbak K, Kjeldsen MK, Olsen KE. Binary toxin and death after Clostridium difficile infection. Emerg Infect Dis. 2011;17(6):976-82. Pubmed Central PMCID: 3358205

42. Barbut F, Decre D, Lalande V, Burghoffer B, Noussair L, Gigandon A, et al. Clinical features of Clostridium difficile-associated diarrhoea due to binary toxin (actin-specific ADP-ribosyltransferase)-producing strains. J Med Microbiol. 2005;54(Pt 2):181-5.

43. Barketi-Klai A, Monot M, Hoys S, Lambert-Bordes S, Kuehne SA, Minton N, et al. The flagellin FliC of Clostridium difficile is responsible for pleiotropic gene regulation during in vivo infection. PLoS One. 2014;9(5):e96876. Pubmed Central PMCID: 4026244.

44. Brouwer MS, Roberts AP, Hussain H, Williams RJ, Allan E, Mullany P. Horizontal gene transfer converts non-toxigenic Clostridium difficile strains into toxin producers. Nat Commun. 2013;4:2601. Pubmed Central PMCID: 3826655.

45. Casey TA, Bosworth BT. Design and evaluation of a multiplex polymerase chain reaction assay for the simultaneous identification of genes for nine different virulence factors associated with Escherichia coli that cause diarrhea and edema disease in swine. J Vet Diagn Investig. 2009;21(1):25-30.

46. Rinttila T, Kassinen A, Malinen E, Krogius L, Palva A. Development of an extensive set of $16 \mathrm{~S}$ rDNA-targeted primers for quantification of pathogenic and indigenous bacteria in faecal samples by real-time PCR. J Appl Microbiol. 2004;97(6):1166-77.

47. Darling AE, Worden P, Chapman TA, Roy Chowdhury P, Charles IG, Djordjevic SP. The genome of Clostridium difficile 5.3. Gut pathogens. 2014; 6(1):4. Pubmed Central PMCID: 4234979

48. Darling AE, Jospin G, Lowe E, Matsen FA, Bik HM, Eisen JA. PhyloSift: phylogenetic analysis of genomes and metagenomes. PeerJ. 2014;2:e243. Pubmed Central PMCID: 3897386.

49. Li H, Handsaker B, Wysoker A, Fennell T, Ruan J, Homer N, et al. The Sequence Alignment/Map format and SAMtools. Bioinformatics. 2009;25(16): 2078-9. Pubmed Central PMCID: 2723002.

50. Schloss PD, Westcott SL, Ryabin T, Hall JR, Hartmann M, Hollister EB, et al. Introducing mothur: open-source, platform-independent, communitysupported software for describing and comparing microbial communities. Appl Environ Microbiol. 2009;75(23):7537-41. Pubmed Central PMCID: 2786419.

51. Bertels F, Silander OK, Pachkov M, Rainey PB, van Nimwegen E. Automated reconstruction of whole-genome phylogenies from short-sequence reads. Mol Biol Evol. 2014;31(5):1077-88. Pubmed Central PMCID: 3995342.

52. Stamatakis A. RAxML-VI-HPC: maximum likelihood-based phylogenetic analyses with thousands of taxa and mixed models. Bioinformatics. 2006; 22(21):2688-90.

53. Lartillot N, Philippe $\mathrm{H}$. A Bayesian mixture model for across-site heterogeneities in the amino-acid replacement process. Mol Biol Evol. 2004; 21(6):1095-109.

54. Stamatakis A, editor. Phylogenetic models of rate heteroginity: a high performance computing perspective. Parallel and Distributed Processing Symposium, 2006. Rhodes Island: IEEE; 2006.

55. Stamatakis A, Hoover $P$, Rougemont J. A rapid bootstrap algorithm for the RAxML Web servers. Syst Biol. 2008;57(5):758-71.

56. Aziz RK, Bartels D, Best AA, DeJongh M, Disz T, Edwards RA, et al. The RAST Server: rapid annotations using subsystems technology. BMC Genomics. 2008;9:75. Pubmed Central PMCID: 2265698

57. Overbeek R, Olson R, Pusch GD, Olsen GJ, Davis JJ, Disz T, et al. The SEED and the Rapid Annotation of microbial genomes using Subsystems Technology (RAST). Nucleic Acids Res. 2014;42(Database issue):D206-14. Pubmed Central PMCID: 3965101

58. Salichos L, Rokas A. Evaluating ortholog prediction algorithms in a yeast model clade. PLoS One. 2011;6(4):e18755. Pubmed Central PMCID: 3076445.

59. Frith MC, Hamada M, Horton P. Parameters for accurate genome alignment. BMC Bioinformatics. 2010;11:80. Pubmed Central PMCID: 2829014.

60. Frith MC, Wan R, Horton P. Incorporating sequence quality data into alignment improves DNA read mapping. Nucleic Acids Res. 2010;38(7):e100. Pubmed Central PMCID: 2853142.
61. Kielbasa SM, Wan R, Sato K, Horton P, Frith MC. Adaptive seeds tame genomic sequence comparison. Genome Res. 2011;21(3):487-93. Pubmed Central PMCID: 3044862

62. Blodel VD, Guillaume JL, Lambiotte R, Lefebvre E. Fast unfolding of communities in large networks. J Stat Mech. 2008:P10008:P10008.

63. Darling AE, Treangen TJ, Messeguer X, Perna NT. Analyzing patterns of microbial evolution using the mauve genome alignment system. Methods Mol Biol. 2007;396:135-52.

64. Rissman Al, Mau B, Biehl BS, Darling AE, Glasner JD, Perna NT. Reordering contigs of draft genomes using the Mauve aligner. Bioinformatics. 2009; 25(16):2071-3. Pubmed Central PMCID: 2723005

65. Sullivan MJ, Petty NK, Beatson SA. Easyfig: a genome comparison visualizer. Bioinformatics. 2011;27(7):1009-10. Pubmed Central PMCID: 3065679.

66. Zhou Y, Liang Y, Lynch KH, Dennis JJ, Wishart DS. PHAST: a fast phage search tool. Nucleic Acids Res. 2011;39(Web Server issue):W347-52. Pubmed Central PMCID: 3125810.

67. Goh S, Chang BJ, Riley TV. Effect of phage infection on toxin production by Clostridium difficile. J Med Microbiol. 2005;54(Pt 2):129-35.

68. Goh S, Ong PF, Song KP, Riley TV, Chang BJ. The complete genome sequence of Clostridium difficile phage phiC2 and comparisons to phiCD119 and inducible prophages of CD630. Microbiology. 2007;153(Pt 3): 676-85.

69. Horgan M, O'Sullivan O, Coffey A, Fitzgerald GF, van Sinderen D, McAuliffe $\mathrm{O}$, et al. Genome analysis of the Clostridium difficile phage PhiCD6356, a temperate phage of the Siphoviridae family. Gene. 2010; 462(1-2):34-43.

70. Cafardi V, Biagini M, Martinelli M, Leuzzi R, Rubino JT, Cantini F, et al. Identification of a novel zinc metalloprotease through a global analysis of Clostridium difficile extracellular proteins. PLoS One. 2013;8(11):e81306. Pubmed Central PMCID: 3841139.

71. Pettit LJ, Browne HP, Yu L, Smits WK, Fagan RP, Barquist $L$, et al. Functional genomics reveals that Clostridium difficile Spo0A coordinates sporulation, virulence and metabolism. BMC Genomics. 2014:15:160. Pubmed Central PMCID: 4028888.

72. Hennequin C, Porcheray F, Waligora-Dupriet A, Collignon A, Barc M, Bourlioux P, et al. GroEL (Hsp60) of Clostridium difficile is involved in cell adherence. Microbiology. 2001;147(Pt 1):87-96.

73. Merrigan MM, Venugopal A, Roxas JL, Anwar F, Mallozzi MJ, Roxas BA, et al. Surface-layer protein A (SIpA) is a major contributor to host-cell adherence of Clostridium difficile. PLoS One. 2013;8(11):e78404. Pubmed Central PMCID: 3827033.

74. Baban ST, Kuehne SA, Barketi-Klai A, Cartman ST, Kelly ML, Hardie KR, et al. The role of flagella in Clostridium difficile pathogenesis: comparison between a non-epidemic and an epidemic strain. PLoS One. 2013;8(9): e73026. Pubmed Central PMCID: 3781105

75. Rousseau C, Poilane I, De Pontual L, Maherault AC, Le Monnier A Collignon A. Clostridium difficile carriage in healthy infants in the community: a potential reservoir for pathogenic strains. Clin Infect Dis. 2012;55(9):1209-15.

76. Dawson LF, Valiente E, Faulds-Pain A, Donahue EH, Wren BW. Characterisation of Clostridium difficile biofilm formation, a role for Spo0A. PLoS One. 2012;7(12):e50527. Pubmed Central PMCID: 3517584

77. Deakin LJ, Clare S, Fagan RP, Dawson LF, Pickard DJ, West MR, et al. The Clostridium difficile spo0A gene is a persistence and transmission factor. Infect Immun. 2012;80(8):2704-11. Pubmed Central PMCID: 3434595.

78. Ethapa T, Leuzzi R, Ng YK, Baban ST, Adamo R, Kuehne SA, et al. Multiple factors modulate biofilm formation by the anaerobic pathogen Clostridium difficile. J Bacteriol. 2013;195(3):545-55. Pubmed Central PMCID: 3554014

79. Petrovski S, Dyson ZA, Seviour RJ, Tillett D. Small but sufficient: the Rhodococcus phage RRH1 has the smallest known Siphoviridae genome at 14.2 kilobases. J Virol. 2012;86(1):358-63. Pubmed Central PMCID: 3255915.

80. Sekulovic O, Garneau JR, Neron A, Fortier LC. Characterization of temperate phages infecting Clostridium difficile isolates of human and animal origins. Appl Environ Microbiol. 2014;80(8):2555-63. Pubmed Central PMCID: 3993186. 